\title{
Mitochondrial DNA variant interactions modify breast cancer risk
}

\author{
Daniel Covarrubias $\cdot$ Ren-Kui Bai · \\ Lee-Jun C. Wong $\cdot$ Suzanne M. Leal
}

Received: 8 May 2008/Accepted: 23 July 2008/Published online: 19 August 2008

(C) The Japan Society of Human Genetics and Springer 2008

\begin{abstract}
Interactions between mitochondrial deoxyribonucleic acid (mtDNA) variants and the risk of developing breast cancer were investigated using DNA samples collected from non-Jewish European American breast cancer patients and ethnically age-matched female controls. Logistic regression was used to evaluate two-way interactions between $17 \mathrm{mtDNA}$ variants. To control for multiple testing, empirical $P$ values were calculated using permutation. Odds ratios (ORs) and corresponding 95\% confidence intervals (CIs) were calculated to measure the contribution of variants in modifying the risk of developing breast cancer. A highly significant interaction was identified between variants $12308 \mathrm{G}$ and $10398 \mathrm{G}$ (empirical $P$ value $=0.0028$, with results suggesting these variants increase the risk of a woman developing breast cancer $(\mathrm{OR}=3.03 ; 95 \%$ CI 1.53-6.11). Nominal significant $P$ values were also observed for interactions between mtDNA variants $709 \mathrm{~A}$ and $16189 \mathrm{C}$; $4216 \mathrm{C}$ and $10398 \mathrm{G} ; 4216 \mathrm{C}$ and 16189C; $10398 \mathrm{G}$ and $16159 \mathrm{C} ; 13368 \mathrm{~A}$ and 16189C; and $14766 \mathrm{~T}$ and 16519C. However, after adjusting for multiple testing, the $P$ values did not remain significant. Although it is important to elucidate the main effect of mtDNA variants on the risk of developing breast cancer,
\end{abstract}

D. Covarrubias and R.-K. Bai contributed equally to this article.

D. Covarrubias · R.-K. Bai · L.-J. C. Wong · S. M. Leal $(\bowtie)$

Department of Molecular and Human Genetics,

Baylor College of Medicine, One Baylor Plaza,

NAB 2015, Houston, TX 77030, USA

e-mail: sleal@bcm.edu

D. Covarrubias

Department of Statistics, Rice University,

Houston, TX 77005, USA understanding gene $\times$ gene interactions will give a greater knowledge of disease etiology and aid in interpreting a woman's risk of developing breast cancer.

Keywords Mitochondrial DNA - Breast cancer . Interaction

\section{Introduction}

Breast cancer is the most diagnosed cancer in women and has the second highest mortality rate among cancers in women. Both environmental (e.g., hormone replacement therapy, age at menopause, obesity, etc.) and genetic factors influence a woman's risk of developing breast cancer. Established high-risk cancer genes BRCA1, BRCA2, PTEN, TP53, LKB1/STK11, CDH1 and low-to-moderate risk cancer genes CHEK2, TGF $\beta 1, C A S P 8, A T M$ account for $25 \%$ of familial and $5-10 \%$ of breast cancer cases within the general population (Antoniou and Easton 2006; LevyLahad and Friedman 2007).

Mitochondria contain their own genome, mitochondrial deoxyribonucleic acid (mtDNA), which is a $16.5-\mathrm{kb}$ circular double-stranded DNA (dsDNA) molecule containing 37 genes. MtDNA is multicopy and maternally inherited. The most prominent roles of mitochondria are production of adenosine triphosphate (ATP) and regulation of cellular metabolism. Mutations within the mtDNA are known to cause various disease phenotypes, such as sensorineural hearing impairment (Forli et al. 2007; Kokotas et al. 2007), Leber's hereditary optic neuropathy (Yen et al. 2006), mitochondrial myopathy (Wong 2007), and mitochondrial encephalopathy, lactacidosis, and stroke-like episodes syndrome (MELAS) (Filosto et al. 2007; Finsterer 2007). Recently, mtDNA variants have been implicated in various 
age-related disorders and complex diseases such as Parkinson's disease (Mortiboys et al. 2007; Onyango 2008), Alzheimer's disease (Carrieri et al. 2001), and several cancers (Petros et al. 2005). Due to its haploid nature and lack of recombination, it is possible to classify individuals through specific mtDNA variants that make up haplogroups. These haplogroups have been shown to possess both geographical-region- and ethnic-specific differences in prevalence. For example, Sub-Saharan Africans mainly carry the L haplogroup, whereas haplogroups $\mathrm{H}, \mathrm{I}, \mathrm{J}$, and $\mathrm{K}$ are predominant in the European populations, with Ashkenazi Jews more frequently carrying the $\mathrm{K}$ haplogroup compared with non-Jewish Europeans (Torroni et al. 1996). This study investigates the role of interaction between mtDNA variants on the risk of developing breast cancer.

Recent studies provide evidence that germline mtDNA variants T3197C, G13708A, G9055GA, T16519C, and A10398G and/or functional variants in linkage disequilibrium (LD) with these variants modify a woman's risk of developing breast cancer (Canter et al. 2005; Bai et al. 2007). In African American women, it was shown that 10398A increases the risk of developing breast cancer (Canter et al. 2005), whereas in European American women, 10398G increases the risk of developing breast cancer (Bai et al. 2007). Additionally, there is evidence that mtDNA variants T3197C, G13708A, G9055A, and T16519C modify breast cancer risk in European American women (Bai et al. 2007). Although it is important to understand the main effects of mtDNA variants on breast cancer risk, knowledge of how mtDNA variants interact gives greater insight into breast cancer etiology and aids in risk assessment. We investigated interactions between mtDNA variants in a case-control study of non-Jewish European American women. Seven significant mtDNA interactions were detected, with one interaction (12308G:10398G; empirical $P$ value $=0.0028$ ) remaining significant after adjusting for multiple testing.

\section{Materials and methods}

Sixty-nine mtDNA variants were genotyped in DNA samples from 156 non-Jewish European American breast cancer patients and 260 ethnically age matched female controls. Of these $69 \mathrm{mtDNA}$ variants, 19 were polymorphic and had minor allele frequencies of $\geq 0.05$. Two of these variants, A4917G and A15607G, were correlated $\left(r^{2}>0.8\right)$ with mtDNA variant G13368A and were not included in the logistic regression analysis. The following 17 mtDNA variants were evaluated for all possible twoway interactions: G709A, G1719A, T3197C, T4216C, G5460A， C7028T， G8251A， G9055A，A10398G, A12308G, G13368A, G13708A, C14766T, A15924G,
C16069T, T16189C, and T16519C. Additional details on subject ascertainment, mtDNA genotyping, and initial analysis can be found in Bai et al. (2007).

Two-way interactions were evaluated using logistic regression by modeling the main effects of two mtDNA variants and the corresponding interaction term

$\log \frac{\theta(\mathbf{x})}{1-\theta(\mathbf{x})}=\beta_{0}+\beta_{1} x_{1}+\beta_{2} x_{2}+\beta_{3}\left(x_{1} * x_{2}\right)$,

where $\theta(\mathbf{x})=P($ Case $\mid \mathbf{x})$.

A total of 136 logistic regression models were evaluated. The common allele within this European American sample was used as the reference allele. Significance was evaluated for the interaction term $\left(\mathrm{H}_{0}\right.$ : $\beta_{3}=0$; the interaction had no effect, versus $H_{1}: \beta_{3} \neq 0$; the interaction had an effect) using the Wald test, as well as for the complete model $\left(\mathrm{H}_{0}: \beta_{1}=\beta_{2}=\beta_{3}=0\right.$; the mtDNA variants and their interaction had no effect, versus $H_{1}: \beta_{i} \neq 0$ for an $i=1,2,3$; one or more of the mtDNA variants and/or their interaction had an effect) using the likelihood ratio test. Due to the large number of tests carried out, the family-wise error rate (FWER) was controlled by calculating empirical $P$ values using 10,000 permutations. Odds ratios (ORs) and 95\% confidence intervals (CIs) were estimated for the mtDNA variant main effects and pair-wise interactions.

\section{Results}

Table 1 summarizes the allelic frequencies for the 17 mtDNA variants found in the DNA samples from cases and controls. Logistic regression analyses revealed seven nominally significant interactions among the variants: 709A and 16189C; 4216C and 10398G; 4216C and 16189C; $12308 \mathrm{G}$ and $10398 \mathrm{G} ; 10398 \mathrm{G}$ and $16519 \mathrm{C} ; 13368 \mathrm{~A}$ and $16189 \mathrm{C}$; and 14766T and 16519C (Table 2). After controlling the FWER, the only interaction term that remained statistically significant was between $10398 \mathrm{G}$ and $12308 \mathrm{G}$ (permuted $P$ value $0.0028)$. Individual analyses of these two variants using logistic regression analysis (Table 3 ) revealed A10398G had a statistically significant main effect (logistic regression $P=0.011)$ and increased a woman's risk of developing breast cancer $\left(\mathrm{OR}=\mathrm{e}^{0.588}=1.8 ; 95 \%\right.$ CI $\left.1.14-2.81\right)$ (Table 3). The main effect of variant A12308G was not significant (logistic regression $P=0.84$ ) (Table 3). Using the complete model with an interaction term (Eq. 1), the logistic regression results suggest women who have both the $10398 \mathrm{G}$ and $12308 \mathrm{G}$ variants had an increased risk of developing breast cancer $\left(\mathrm{OR}=\mathrm{e}^{-0.771-0.006+1.887}=3.03\right.$; 95\% CI 1.53-6.11) (Table 4). Whereas women who carry the $10398 \mathrm{~A}$ variant and the $12308 \mathrm{G}$ variant had a decreased risk of developing breast cancer $\left(\mathrm{OR}=\mathrm{e}^{-0.771}=0.46\right.$; 
Table 1 Summary of the 17 mitochondrial deoxyribonucleic acid (mtDNA) variants included in the analysis

\begin{tabular}{|c|c|c|c|c|c|}
\hline \multirow[t]{2}{*}{ SNPs } & \multicolumn{2}{|c|}{ Cases $(n=156)$} & \multicolumn{2}{|c|}{ Controls $(n=260)$} & \multirow[t]{2}{*}{ Odds ratio } \\
\hline & Positive & $\%$ & Positive & $\%$ & \\
\hline G709A & 20 & 12.82 & 25 & 9.62 & 1.39 \\
\hline G1719A & 17 & 10.90 & 18 & 6.92 & 1.64 \\
\hline Т3197C & 6 & 3.85 & 31 & 11.92 & 0.31 \\
\hline T3394C & 3 & 1.92 & 4 & 1.54 & 1.30 \\
\hline T4216C & 26 & 16.67 & 52 & 20.00 & 0.81 \\
\hline G5460A & 4 & 2.56 & 18 & 6.92 & 0.38 \\
\hline C7028T & 91 & 58.33 & 149 & 57.31 & 1.04 \\
\hline G8251A & 16 & 10.26 & 16 & 6.15 & 1.74 \\
\hline G9055A & 29 & 18.59 & 18 & 6.92 & 3.03 \\
\hline A10398G & 50 & 32.05 & 54 & 20.77 & 1.79 \\
\hline A12308G & 41 & 26.28 & 66 & 25.38 & 1.05 \\
\hline G13708A & 12 & 7.69 & 40 & 15.38 & 0.47 \\
\hline $\mathrm{C} 14766 \mathrm{~T}$ & 84 & 53.85 & 137 & 52.69 & 1.04 \\
\hline A15924G & 15 & 9.62 & 16 & 6.15 & 1.62 \\
\hline $\mathrm{C} 16069 \mathrm{~T}$ & 10 & 6.41 & 30 & 11.54 & 0.54 \\
\hline T16189C & 21 & 13.46 & 35 & 13.46 & 1.01 \\
\hline T16519C & 122 & 78.21 & 167 & 64.23 & 1.98 \\
\hline
\end{tabular}

Displayed in the table are the mtDNA variant name, the number of variants present in cases and controls, and the percentage of cases and controls with the variant

SNPs single nucleotide polymorphisms

Table 2 Significant two-way interactions

\begin{tabular}{llll}
\hline Interaction & $\begin{array}{l}\text { Logistic } \\
\text { interaction } P \\
\text { value }\end{array}$ & $\begin{array}{l}\text { Adjusted } \\
\text { interaction } P \\
\text { value }\end{array}$ & $\begin{array}{l}\text { Logistic } \\
\text { regression global } \\
P \text { value }\end{array}$ \\
\hline 709A:16189C & 0.0081 & 0.1102 & 0.0235 \\
4216C:10398G & 0.0079 & 0.1070 & 0.0009 \\
4216C:16189C & 0.0150 & 0.2190 & 0.0752 \\
12308G:10398G & 0.0004 & 0.0028 & 0.0002 \\
10398G:16519C & 0.0221 & 0.3289 & 0.0001 \\
13368A:16189C & 0.0099 & 0.1392 & 0.0440 \\
14766T:16519C & 0.0158 & 0.2304 & 0.0016 \\
\hline
\end{tabular}

Displayed in the table are the names of the mtDNA variants, nominal interaction $P$ values $\left(H_{0}: \beta_{3}=0 ; H_{1}: \beta_{3} \neq 0\right)$, interaction $P$ values adjusted for the family-wise error rate (FWER) and nominal global $P$ values $\left(H_{0}: \beta_{1}=\beta_{2}=\beta_{3}=0 ; H_{1}: \beta_{i} \neq 0\right.$ for one or more $i=1,2$, 3) obtained from analysis of deviance

95\% CI 0.24-0.88) (Table 4). Additionally, in the presence of variant $12308 \mathrm{~A}$, variant $10398 \mathrm{G}$ had no effect on breast cancer risk $\left(\mathrm{OR}=\mathrm{e}^{-0.006}=0.99, P=0.98 ; 95 \%\right.$ CI 0.56 1.77) (Table 4).

Variants $10398 \mathrm{~A}$ and $12308 \mathrm{~A}$ define the $\mathrm{T}$ haplogroup along with variants $4216 \mathrm{C}, 7028 \mathrm{~T}, 13368 \mathrm{~A}$, and $15607 \mathrm{G}$. Variants 10398A and 12308G define the U haplogroup along with the variant $9055 \mathrm{G}$. Although belonging to the T haplogroup does not influence a woman's risk of developing breast cancer, membership in the U haplogroup has been shown to greatly reduce a woman's risk of developing breast cancer $[\mathrm{OR}=0.38 ; P$ value $=0.03$ (adjusted for multiple testing)] (Bai et al. 2007). In this analysis, it can be seen that regardless of G9055A status, a woman carrying the mtDNA variants $12308 \mathrm{G}$ and $10398 \mathrm{~A}$ has a reduced risk of developing breast cancer $(\mathrm{OR}=$ $\mathrm{e}^{-0.771}=0.46 ; 95 \%$ CI 0.24-0.88) (Table 4).

\section{Discussion}

The 10398A variant has previously been implicated in increasing the risk of developing both breast and prostate cancer in African Americans (Canter et al. 2005; Mims et al. 2006) and breast and esophageal cancer in Indians (Darvishi et al. 2007), whereas the 10398G variant increases the risk of developing breast cancer in European American women (Bai et al. 2007). Two independent studies could not replicate the findings that the 10398A variant increases the risk of developing breast cancer in African American women (Setiawan et al. 2008) or that the 10398G variant increases the risk of developing breast cancer in women from Spain and the Canary Islands (Mosquera-Miguel et al. 2008).

One of the interactions, $4216 \mathrm{C}$ and $10398 \mathrm{G}$, observed in this study although not statistically significant after controlling the FWER (Table 2), was previously reported in the Canter et al. (2005) case-control study of African American breast cancer patients (Verma et al. 2007). In the Canter study, a significant main effect was observed for 10398A, which increased a woman's risk of developing breast cancer $(\mathrm{OR}=1.6)$, whereas no statistically significant main effect was observed for T4216C and a synergistic interaction was observed between $4216 \mathrm{C}$ and 10398A, where the presence of both variants increased a woman's risk of developing breast cancer $(\mathrm{OR}=3.1)$ (Verma et al. 2007). In this case-control study of European Americans, when each variant was analyzed independently, an increased risk was observed for 10398G $\left(\mathrm{OR}=\mathrm{e}^{0.588}=1.8 ; P=0.01\right)$ (Table 3$)$, and there was also no statistically significant main effect observed for T4216C variant $(P=0.39)$ (data not shown). This study observed a nominally significant interaction between 4216C and 10398G $(P=0.008)$, which did not remain significant after controlling the FWER $(P=0.11)$ (Table 2). Although women having the 4216T and 10398G variants had an increase in developing breast cancer $\left(\mathrm{OR}=\mathrm{e}^{1.106}=3.02 ; 95 \%\right.$ CI 1.72-5.32), women with the 4216C and 10398G variants had a decreased risk of developing breast cancer $\left(\mathrm{OR}=\mathrm{e}^{0.191+1.106-1.492}=0.82\right.$; 
Table 3 Coefficient estimates for mitochondrial deoxyribonucleic acid (mtDNA) variants A10398G and A12308G

\begin{tabular}{|c|c|c|c|c|c|c|}
\hline \multirow[t]{2}{*}{ Parameters } & \multirow[t]{2}{*}{ Variant(s) } & \multicolumn{3}{|c|}{ Coefficient estimate (standard error) } & \multirow[t]{2}{*}{ Parameter $P$ value } & \multirow[t]{2}{*}{ Global $P$ value } \\
\hline & & $A 12308 G$ & A10398G & Interaction & & \\
\hline$\beta_{1}$ & $12308 \mathrm{G}^{\mathrm{a}}$ & $0.047(0.231)$ & - & - & 0.839 & 0.841 \\
\hline$\beta_{2}$ & $10398 G^{b}$ & - & $0.588(0.230)$ & - & 0.011 & 0.011 \\
\hline$\beta_{1}+\beta_{2}$ & $12308 \mathrm{G}$ and $10398 \mathrm{G}^{\mathrm{c}}$ & $-0.072(0.238)$ & $0.602(0.235)$ & - & $0.762,0.010^{\mathrm{e}}$ & 0.037 \\
\hline$\beta_{1}+\beta_{2}+\beta_{3}$ & 12308G:10398G ${ }^{\mathrm{d}}$ & $-0.771(0.329)$ & $-0.006(0.294)$ & $1.887(0.53)$ & $0.0004^{\mathrm{f}}$ & 0.0005 \\
\hline
\end{tabular}

Analyses were carried out including only A12308G in the model; including only A10398G in the model; including A12308G and A10398G in the model without an interaction term; and including A12308G, A10398G with an interaction term in the model. Displayed in the table are the parameters, names of variants included in the logistic regression model, coefficient estimates and their standard error, parameter $P$ values (Wald test), and global $P$ values (logistic regression) obtained from analysis of deviance

${ }^{a}$ Only A12308G included in the logistic regression model

b Only A10398G included in the logistic regression model

c Variants A10398G and A12308G included in the model, no interaction term included in the logistic regression model

d Variants A10398G and A12308G included in model with an interaction term included in the logistic regression model

e $P$ values for $\beta_{1}$ and $\beta_{2}$, respectively

f $P$ value for $\beta_{3}$ only

Table 4 Summary of results from the logistic regression for mitochondrial deoxyribonucleic acid (mtDNA) variants A10398G and A12308G

\begin{tabular}{llllll}
\hline Parameter & Variant & $\hat{\beta}_{i}$ (coef. est.) & Standard error & $Z$ value & Parameter $P$ value \\
\hline$\beta_{0}$ & - & -0.052 & 0.132 & -3.964 & $7.39 \mathrm{e}-05$ \\
$\beta_{1}$ & $12308 \mathrm{G}$ & -0.771 & 0.329 & -2.343 & 0.019 \\
$\beta_{2}$ & $10398 \mathrm{G}$ & -0.006 & 0.294 & -0.022 & 0.983 \\
$\beta_{3}$ & $12308 \mathrm{G}: 10398 \mathrm{G}$ & 1.887 & 0.530 & 3.559 & 0.0004 \\
\hline
\end{tabular}

Displayed in the table are the parameters, names of variants, estimates of the $\beta$ coefficients, estimates of the standard error, $Z$ values, and parameter $P$ values

Table 5 Summary of results from the logistic regression for mitochondrial deoxyribonucleic acid (mtDNA) variants T4216C and A10398G

\begin{tabular}{llllll}
\hline Parameter & Variant & $\hat{\beta}_{i}$ (coefficient estimate) & Standard error & $Z$ value & Parameter $P$ value \\
\hline$\beta_{0}$ & - & -0.688 & 0.128 & -5.381 & $7.41 \mathrm{e}-08$ \\
$\beta_{1}$ & $4216 \mathrm{C}$ & 0.191 & 0.362 & 0.528 & 0.598 \\
$\beta_{2}$ & $10398 \mathrm{G}$ & 1.106 & 0.288 & 3.849 & 0.0001 \\
$\beta_{3}$ & $4216 \mathrm{C}: 10398 \mathrm{G}$ & -1.492 & 0.561 & -2.657 & 0.008 \\
\hline
\end{tabular}

Shown in the table are parameters, variant names, $\beta$ coefficient estimates, standard error estimates, $Z$ values, and parameter $P$ values

95\% CI 0.39-1.67) (Table 5). For variant A10398G, the Canter study (2005) used 10398G as the reference group, because it is the common allele in African Americans. In European Americans, 10398A is the common allele and was used as the reference group in this study. When the same reference group (10398G) was used as in the Canter study in the logistic regression model, women who had the 4216C and 10398A variants had a decreased risk of developing breast cancer $\left(\mathrm{OR}=\mathrm{e}^{-1.11-1.30+1.492}=0.40\right.$; 95\% CI 0.17-0.93) (data not shown). The differences in direction of the effect between the Canter and this study suggest the functional variants that increase a woman's risk of developing breast cancer are on different haplotypes in African American and European American women.

This study presents strong evidence that genes within the mitochondria interact to increase the risk of breast cancer. It is demonstrated that mtDNA variants A12308G and A10398G located within the MT-TL2 and MT-ND3 genes, respectively, interact, affecting a woman's risk of developing breast cancer. In particular, mtDNA variants 10398G and 12308G interact synergistically, increasing a woman's risk of developing breast cancer. 
Acknowledgments This study was supported in part by NIH grant CA10023 and Department of Defense US Army Breast Cancer Research Program, DAMD17-01-1-0258.

\section{References}

Antoniou AC, Easton DF (2006) Models of genetic susceptibility to breast cancer. Oncogene 25:5898-5905

Bai RK, Leal SM, Covarrubias D, Liu A, Wong LJ (2007) Mitochondrial genetic background modifies breast cancer risk. Cancer Res 67:4687-4694

Canter JA, Kallianpur AR, Parl FF, Millikan RC (2005) Mitochondrial DNA G10398A polymorphism and invasive breast cancer in African-American women. Cancer Res 65:8028-8033

Carrieri G, Bonafe M, De Luca M, Rose G, Varcasia O, Bruni A, Maletta R, Nacmias B, Sorbi S, Corsonello F, Feraco E, Andreev KF, Yashin AI, Franceschi C, De Benedictis G (2001) Mitochondrial DNA haplogroups and APOE4 allele are nonindependent variables in sporadic Alzheimer's disease. Hum Genet 108:194-198

Darvishi K, Sharma S, Bhat AK, Rai E, Bamezai RN (2007) Mitochondrial DNA G10398A polymorphism imparts maternal Haplogroup $\mathrm{N}$ a risk for breast and esophageal cancer. Cancer Lett 249:249-255

Filosto M, Tomelleri G, Tonin P, Scarpelli M, Vattemi G, Rizzuto N, Padovani A, Simonati A (2007) Neuropathology of mitochondrial diseases. Biosci Rep 27:23-30

Finsterer J (2007) Genetic, pathogenetic, and phenotypic implications of the mitochondrial A3243G tRNALeu(UUR) mutation. Acta Neurol Scand 116:1-14

Forli F, Passetti S, Mancuso M, Seccia V, Siciliano G, Nesti C, Berrettini S (2007) Mitochondrial syndromic sensorineural hearing loss. Biosci Rep 27:113-123

Kokotas H, Petersen MB, Willems PJ (2007) Mitochondrial deafness. Clin Genet 71:379-391
Levy-Lahad E, Friedman E (2007) Cancer risks among BRCA1 and BRCA2 mutation carriers. Br J Cancer 96:11-15

Mims MP, Hayes TG, Zheng S, Leal SM, Frolov A, Ittmann MM, Wheeler TM, Prchal JT (2006) Mitochondrial DNA G10398A polymorphism and invasive breast cancer in African-American women. Cancer Res 66:1880; author reply 1880-1881

Mortiboys HJ, Schaefer J, Reichmann H, Jackson S (2007) Mitochondrial dysfunction in Parkinson's disease-revisited. Neurol Neurochir Pol 41:150-159

Mosquera-Miguel A, Alvarez-Iglesias V, Carracedo A, Salas A, Vega A, Milne R, de Leon AC, Benitez J (2008) Is mitochondrial DNA variation associated with sporadic breast cancer risk? Cancer Res 68:623-625 author reply 624

Onyango IG (2008) Mitochondrial dysfunction and oxidative stress in Parkinson's disease. Neurochem Res 33:589-597

Petros JA, Baumann AK, Ruiz-Pesini E, Amin MB, Sun CQ, Hall J, Lim S, Issa MM, Flanders WD, Hosseini SH, Marshall FF, Wallace DC (2005) mtDNA mutations increase tumorigenicity in prostate cancer. Proc Natl Acad Sci USA 102:719-724

Setiawan VW, Chu LH, John EM, Ding YC, Ingles SA, Bernstein L, Press MF, Ursin G, Haiman CA, Neuhausen SL (2008) Mitochondrial DNA G10398A variant is not associated with breast cancer in African-American women. Cancer Genet Cytogenet 181:16-19

Torroni A, Huoponen K, Francalacci P, Petrozzi M, Morelli L, Scozzari R, Obinu D, Savontaus ML, Wallace DC (1996) Classification of European mtDNAs from an analysis of three European populations. Genetics 144:1835-1850

Verma M, Naviaux RK, Tanaka M, Kumar D, Franceschi C, Singh KK (2007) Meeting report: mitochondrial DNA and cancer epidemiology. Cancer Res 67:437-439

Wong LJ (2007) Pathogenic mitochondrial DNA mutations in protein-coding genes. Muscle Nerve 36:279-293

Yen MY, Wang AG, Wei YH (2006) Leber's hereditary optic neuropathy: a multifactorial disease. Prog Retin Eye Res 25:381-396 